\title{
Design of three-component one-dimensional photonic crystals for alteration of optical contrast and omni-directional reflection
}

\author{
Anna V. Baldycheva ${ }^{\mathrm{a}}$, Vladimir A. Tolmachev ${ }^{\mathrm{b}}$, Tatiana S. Perova ${ }^{* a}$ and Kevin Berwick ${ }^{\mathrm{c}}$ \\ ${ }^{a}$ Department of Electronic and Electrical Engineering, Trinity College Dublin, Dublin 2, Ireland; \\ ${ }^{b}$ Ioffe Physical Technical Institute, Polytechnicheskaya 26, St. Petersburg, Russia; \\ ${ }^{c}$ Department of Electronic and Communications Engineering, Dublin Institute of Technology, Kevin \\ St, Dublin 8, Ireland
}

\begin{abstract}
In this study, three-component One-Dimensional (1D) Photonic Crystal (PC) structures were investigated by modeling them as two-component PCs with an additional regular layer. The Gap Map approach and the Transfer Matrix Method were used in order to mathematically describe these structures. The introduction of a third component to a 1D PC allows manipulation of the optical contrast to a high degree of precision by varying the thickness and refractive index of the additional layer. It also partially reduces the area of the photonic band gaps (PBGs) on the gap map, leaving the remainder of the PBG area unchanged from that of the gap map for the original, two-component, PC. Using this approach to decrease the optical contrast in photonic crystals allows omni-directional bands to be obtained in highcontrast periodic structures constructed from, for example, an array of silicon and air.
\end{abstract}

Keywords: 1D Photonic Crystal, Tunable Photonic Crystal, Multi-component Photonic Crystal, Photonic Band Gap, Photonic Gap Map

\section{INTRODUCTION}

Photonic Crystals (PCs) ${ }^{1,2}$ have attracted considerable scientific and commercial interest in recent decades because of their unique properties in manipulating light. Photonic Crystals exhibit total reflection over a range of frequencies, that is, they have a Photonic Band Gap (PBG). Optical devices based on multi-dimensional PCs can be used to fabricate numerous optical elements, including waveguides, reflectors, attenuators of spontaneous atomic emission and devices for controlling emissions in micro-cavities ${ }^{1-3}$. These devices operate by the PBG effect and, therefore, possess high sensitivity to the refractive indices, $N$, of their components. Manipulation of $N$ allows the design of PBG materials whose optical characteristics can be varied over a wide range. The PCs are normally composed of two-components, with high $\left(N_{H}\right)$ and low $\left(N_{L}\right)$ refractive indices. By changing the value of $N_{H}$ or $N_{L}$, tuning of the PBG position can be achieved. One of the most promising high-refractive indexes, $(H)$ materials is $\mathrm{Si}$, due to its low-cost and ready availability. However, the variation of the refractive index, $N_{H}$, of Si by heating, or free carrier generation, is minimal $\left(\Delta N_{S i}=0.001\right)$. At the same time, variation of the low-refractive index component, $N_{L}$, has a far greater impact on the PBG shift. In order to change the value of $N_{L}$, i.e. to decrease or increase the optical contrast, the air-gaps, or channels, can be impregnated with various liquid or solid fillers. A number of studies were published recently on the infiltration of air-channels with Liquid Crystals (LCs), which enables tuning of the optical contrast due to reorientation of the LC molecules ${ }^{4}$. Typical liquid crystals used for device applications have refractive indices in the range 1.5 to 1.8. Although this technique is technologically well developed, there is an alternative approach to engineering the PBG.

*perovat@tcd.ie; phone 3531 896-1432; fax 3531 677-2442; http://www.mee.tcd.ie

Photonic Crystal Materials and Devices IX, edited by Hernán R. Míguez, Sergei G. Romanov,

Lucio Claudio Andreani, Christian Seassal, Proc. of SPIE Vol. 7713, 771321

(c) 2010 SPIE · CCC code: 0277-786X/10/\$18 - doi: 10.1117/12.854977

Proc. of SPIE Vol. $7713771321-1$ 
This approach requires the introduction of an additional component to the PC, forming a three-component PC. It is worthwhile to refer to recent investigations of multi-component, 1D and 2D PCs ${ }^{5,6}$. The authors of Ref. ${ }^{6}$ have shown, based on the example of 2D PCs, a shift of the gaps' edges. This shift depends on the thickness and dielectric constant of the intermediate layer on the surface of the cylindrical pores. They explained that the shift is associated with a decrease in the effective dielectric constant of the system. The authors of another paper ${ }^{7}$ studied a multi-component model for a more complex, 3D PC structure, and have shown, based on an opal structure sample, that it is possible to selectively manipulate the PBGs in this PC. They have demonstrated the possibility of controlling the emergence of the PBG, along with the width and spectral shift, and also digital ON/OFF switching, by varying the additional layer's thickness, permittivity and other parameters in the multi-component PC.

In our paper ${ }^{8}$, we selected the 1D PC as the simplest example for calculations, as well as for demonstrating PBG modification, in both position and width, due to the introduction of an additional regular $t$-layer. We have analysed the properties of a three-component PC structure based on a Gap Map (GM) approach ${ }^{3}$. As demonstrated earlier, the GM method is a very efficient approach for the analysis of both tunable ${ }^{9}$ and composite $1 \mathrm{D} \mathrm{PCs}^{10}$, as well as for PC structures with omni-directional bands and defects ${ }^{11}$.

Over the last decade, particular attention has been paid to the development of devices based on one-dimensional PCs, since they possess certain advantages over two- and three-dimensional PCs. These advantages are mainly related to their simpler fabrication, lower cost, and the possibility of creating omni-directional bands (ODBs) ${ }^{12-14}$. Calculations of the optical properties of 1D PCs with ODBs must take into account the refractive index contrast (or optical contrast) $N_{H} / N_{L}$, which should be large if one wants to achieve a maximal PBG width. It should also be noted that photonic structures with an optical contrast that is too large have the disadvantage that closure of the PBGs can occur at particular angles of incidence of light and, therefore, result in the disappearance of the ODBs for the structure. In accordance with ${ }^{3,13}$, the minimum value of $N_{L}$ should be greater than 1.1 for light incident from air. As a result, it is impossible to obtain ODBs for structures with a high-optical contrast, such as grooved Si with contrast 3.42/1 which can be used as a 1D PC material in the infrared (IR) range ${ }^{15}$. At the same time the introduction of the additional layer with a particular optical thickness into high-contrast PC can, under some conditions, results in creation of omni-directional bands. In the present paper, our investigations are focused on PCs with a variety of optical contrasts, as well as for different angles and polarisations of the incident light. Particular attention is paid here to the tuning of the optical contrast in 1D PCs.

\section{RESULTS AND DISCUSSION}

\subsection{Modeling of three-component photonic crystal structure}

A periodic structure with linear grooves on the micro scale ${ }^{16}$ is considered here as a model for a 1D PC structure (Fig.1). This consists of alternating layers of material with high, $N_{H}$, and low, $N_{L}$, refractive indices. The refractive index of Si for the $H$ component in the IR range is chosen as $N_{S i}=N_{H}=3.42$. The refractive index of air for the $L$ component is $N_{\text {air }}=N_{L}=1$. In this case, the refractive index contrast is $\Delta N=N_{H} / N_{L}=3.42 / 1$ and the PC obtained can be considered a highoptical contrast PC. Therefore, only a small number of periods $m$ are required to obtain a PBG with a reflection of greater than 0.999. Let us consider two examples of this 1D PC with the number of periods $m=5$ and $m=10$. We would like to investigate the impact on the PBGs caused by insertion of an additional regular layer such as, for example, $\mathrm{SiO}_{2}$ with a refractive index $N_{t}=1.5$, which can be deposited on the Si walls of the 1D PC structure with a definite lattice constant, $A$. The refractive index of the incoming and outgoing medium, $N$, is taken as $N=1$. All the optical characteristics presented in this study utilize normalized frequency $N F=A / \lambda$ units and, therefore, can be applied to a wide range of structure sizes, including micro- and nano-structures.

Calculations of the reflection and transmission spectra of 1D PCs with a number of lattice periods, $m$, and a filling fraction of the Si walls $f_{S i}=D_{H} / A$, can be performed using the Transfer-Matrix Method (TMM) ${ }^{17}$. This method is attractive due to the ease of introduction of an arbitrary number of additional layers at any point in the TMM's equation (Eqs. (1) and (2)). 

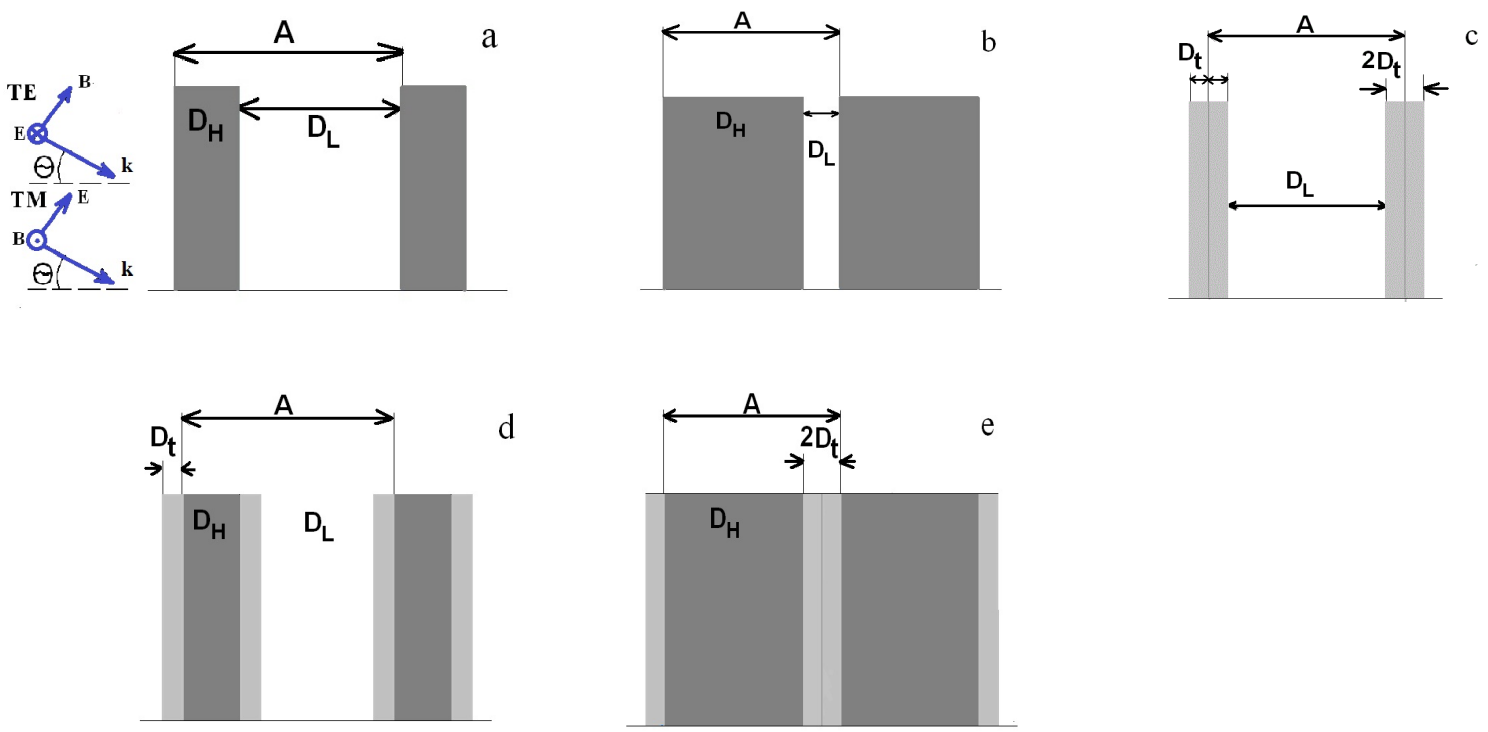

Fig.1. Schematic fragments of $(a, b, c, e)$ two-component 1D PC and $(d)$ three-component 1D PC with fixed value of lattice constant $A$ and an additional $t$-layer. The thicknesses of $H, L$ and $t$ layers are $D_{H}, D_{L}, D_{t}$, accordingly.

The Transfer Matrix, $S_{2}$, used in calculations for a two-component 1D PC structure, is given schematically by:

$$
\mathrm{S}_{2}=\left(\mathrm{M}_{\mathrm{H}} \cdot \mathrm{M}_{\mathrm{L}}\right)^{\mathrm{m}} \cdot \mathrm{M}_{\mathrm{H}}
$$

for a three-component PC matrix, $S_{3}$, as shown in Fig.1d, the matrix is ${ }^{8}$ :

$$
\mathrm{S}_{3}=\left(\mathrm{M}_{\mathrm{t}} \cdot \mathrm{M}_{\mathrm{H}} \cdot \mathrm{M}_{\mathrm{t}} \cdot \mathrm{M}_{\mathrm{L}}\right)^{\mathrm{m}} \cdot \mathrm{M}_{\mathrm{t}} \cdot \mathrm{M}_{\mathrm{H}} \cdot \mathrm{M}_{\mathrm{t}},
$$

where $M_{H}, M_{L}$ and $M_{t}$ are the matrices of the $H, L$ and $t$ layers, respectively.

Indeed, the reflectance spectra obtained for different filling fraction, $f_{S i}$, values can reveal significant differences in PBG formation ${ }^{5}$. As demonstrated earlier, the best analysis of the optical properties of PC structures is obtained using a combination of TMM and GM approaches ${ }^{9,10,18-20}$.

The GM approach enables us to choose the parameters required for the engineering of 1D PCs based on grooved $\mathrm{Si}^{16}$. In particular, we can identify the range of frequencies that can be practically realized for these structures, and, therefore, establish the design criteria for different regions of the IR spectrum.

Table 1. Maximum value of filling fraction $\operatorname{Limf}_{S i}$ for different values of $t$-layer thickness, $D_{t}$, required for GM calculations of a three-component PC with lattice period $A$.

\begin{tabular}{|llllllllll|}
\hline$D_{t}$ & $0.03 \mathrm{~A}$ & $0.07 \mathrm{~A}$ & $0.10 \mathrm{~A}$ & $0.13 \mathrm{~A}$ & $0.20 \mathrm{~A}$ & $0.27 \mathrm{~A}$ & $0.33 \mathrm{~A}$ & $0.40 \mathrm{~A}$ & $0.47 \mathrm{~A}$ \\
\hline Limf $_{S i}$ & 0.94 & 0.86 & 0.80 & 0.74 & 0.60 & 0.46 & 0.34 & 0.20 & 0.06 \\
\hline
\end{tabular}

In order to create a GM of PBGs we need to generate the set of reflection spectra for each value of the filling fraction $f_{S i}=D_{H} / A$ ranging from 0 to 1 . Next, values of frequency satisfying the required criterion for PBG formation $(R>0.999)$ are selected. We assume that the lattice constant $A$ and the $t$-layer thickness, $D_{t}$, do not change, since the $t$-layers with refractive index $N_{t}$ were introduced on both sides of the Si wall $H$ in our PC structure (Fig. 1d). When generating $f_{S i}$, the values of $D_{H}$ for both two-component and the three-component PCs are the same and, therefore, we can compare two different PC structures using a single $f_{S i}$ scale. However, in this case, the value of $D_{L}$ decreases after the introduction of the $t$-layer with thickness $D_{t}$ on both sides of the Si walls $D_{H}$ (Fig.1d). As a result, when plotting the GM, not all values for the filling fraction $f_{S i}$, from 0.01 to 0.99 can be realized in practice. The larger the value of $D_{t}$, the lower the 
maximum value of the filling fraction $f_{S i}$ that can be realized in the three-component PC. In Table 1 , the maximum values of the filling fractions $f_{S i}$, obtained for different values of the $t$-layer thickness $D_{t}$, are presented.

\subsection{Engineering of the optical contrast of a three-component photonic crystal structure}

Let us calculate the GMs of the PBG for a three-component PC with number of periods $m=10$, obtained by the introduction of a $t$-layer with various thicknesses $D_{t}$ (Table 1) into a two-component PC with high optical contrast $N=3.42 / 1$ (Fig 2, Curve 1). For comparison purposes, we will also calculate the GM for the limiting case, when the $L$ component is totally replaced by the $t$-layer and we obtain a two-component PC with medium optical contrast $N=3.42 / 1.5$ (Fig 2 b, curve 1.5).
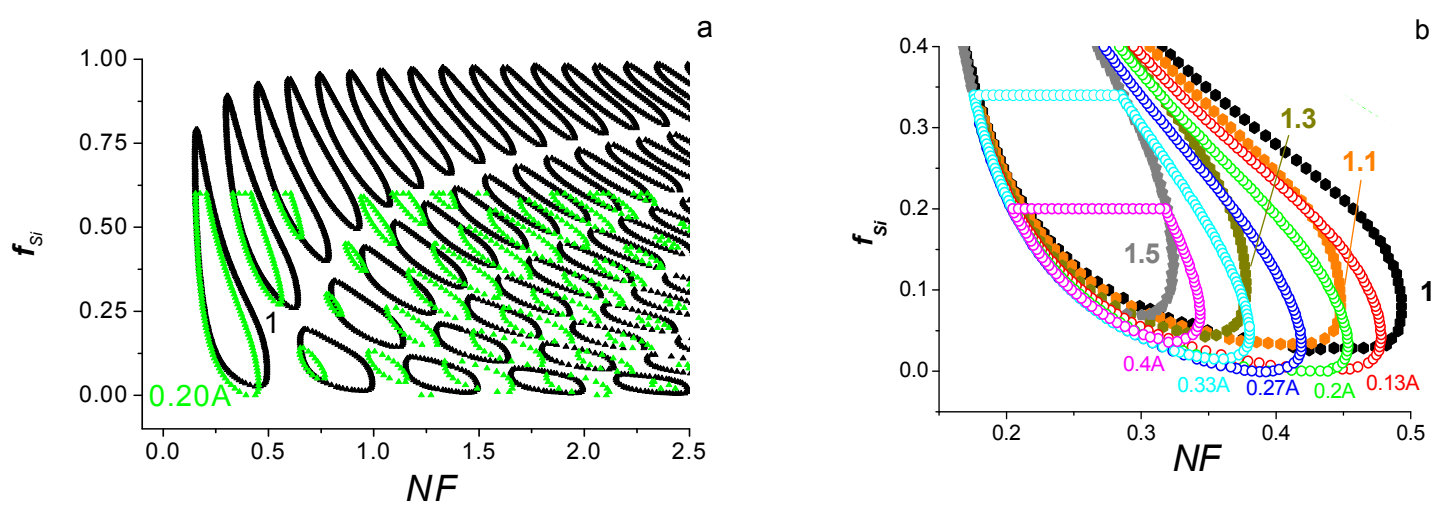

Fig.2. a) The GMs for two-component PC with optical contrasts $N=3.42 / 1$ (1) and for three-component PC with $t$-layer with $N_{\mathrm{t}}=1.5$ and $D_{t}=0.20 \mathrm{~A}(0.20 \mathrm{~A})$ in a wide range of $N F$. b) Comparison of the lowest PBGs for three-component PC with various thicknesses $D_{t}$ and two-component PCs with different optical contrasts $(3.42 / 1,3.42 / 1.1,3.42 / 1.3$ and 3.42/1.5), shown by numbers beside the curves. The normal incidence of light is considered here.

Figure 2a shows that the introduction of a $t$-layer with $D_{t}=0.20 \mathrm{~A}$ and $N_{t}=1.5$ results in i) a decrease in the size of all PBGs and ii) a red shift of the PBGs with respect to the original two-component PC. Similar behavior was observed earlier in the GM of the PBGs of a two-component PC as $N_{L}$ increases ${ }^{9,15}$. Thus, the introduction of the $t$-layer has a similar effect to the substitution of $N_{L}$, i.e. it serves to decrease the optical contrast or, in accordance with Ref. ${ }^{6}$, decrease the effective dielectric constant. As can be seen in Fig. 2a, the size of the high-order PBG regions for the threecomponent PCs differs from that in two-component PCs. In the range of $N F$ values from 0.7 to 1 , a suppression of high order PBGs takes place.

It can be also be seen in Fig. $2 \mathrm{~b}$ that the blue edges of the first PBG, for $N_{L}=1.1$ and 1.3 for the two-component PC, intersect the blue edges for the three-component $\mathrm{PC}$ at $f=0.2$, with $D_{t}=0.13 \mathrm{~A}$ and $0.27 \mathrm{~A}$. Therefore, the addition of the new layer to form the three-component PC has an impact on the PBG of the crystal, demonstrating the possibility of manipulating the optical contrast by changing the thickness of the additional regular layer in the PC structure. In other words, the $D_{t}$ value provides an equivalent value of $N L_{\text {equ }}$ for a two-component PC with the same $N_{H}$ value.

From Fig. 3, for the chosen value of $D_{t}$, the range of $N L_{\text {equ }}$ can be varied from 1.1 to 1.5 (in the limiting case), where the $L$-component is totally replaced by $\mathrm{SiO}_{2}$ and we have a two-component $\mathrm{PC}$ with a contrast of 3.42/1.5. As discussed earlier, modification of the optical contrast is possible by changing the thickness of the additional layer $D_{t}$. Changing the refractive index $N_{t}$ is an alternative method of modifying the optical contrast and, like $D_{t}$, the value of $N_{t}$ can be altered technologically. So, by changing the values of $D_{t} \cdot N_{t}$ for the $t$-layer in the interference structure, new types of PC structures with optical contrasts from $3.42 / 1$ to $3.42 / 1.5$ can be designed.

\subsection{Formation of omni-directional band in a three-component photonic crystal structure}

It is well known that the ODB is normally determined by overlapping of the regions of high reflectivity for both polarisations and all angles, $\theta$, of the incident light ${ }^{3}$. However, it was already shown that an ODB cannot be obtained, 
regardless of filling fraction, for $1 \mathrm{D}$ PC structures with a value of $N_{L}$ less than $1.1^{24}$. As mentioned in the section 2.2, we can easily change the optical contrast of a PC by introducing a third layer. Let us now calculate the transmission spectra for various, three-component, PC structures with different $D_{t}$ values, over a range of possible angles of incidence from $0^{\circ}$ to $85^{\circ}$, with a step size of $5^{\circ}$ (Fig. 4). The width of the PBGs for TE polarization increases with an increase in $\theta$, while for TM polarisation it decreases. As a rule, it is necessary to ensure that the PBGs for TM polarisation have a common

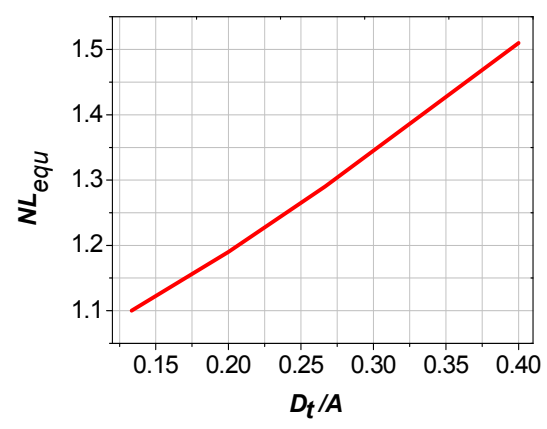

Fig.3. The refractive index of the low component in the equivalent two-component $\mathrm{PC} N L_{\text {equ }} \mathrm{vs}$. the $t$-layer thickness over lattice period $A$ of three-component PC with refractive index $N_{t}=1.5$.

area for different $\theta$ in order for this area to be identified as the ODB. Figure 4 shows $T$ spectra for a three-component PC with various $D_{t}$ and also for the equivalent two-component PC, obtained for TM polarization. Note that we concentrate on the lowest PBGs and, for clarity, only two limiting cases for the incident angle, $\theta=0^{\circ}$ and $\theta=85^{\circ}$, are shown. The common ODB areas are depicted by the rectangles. For comparison, Fig. 4a shows the spectra of a twocomponent PC with a contrast of 3.42/1.
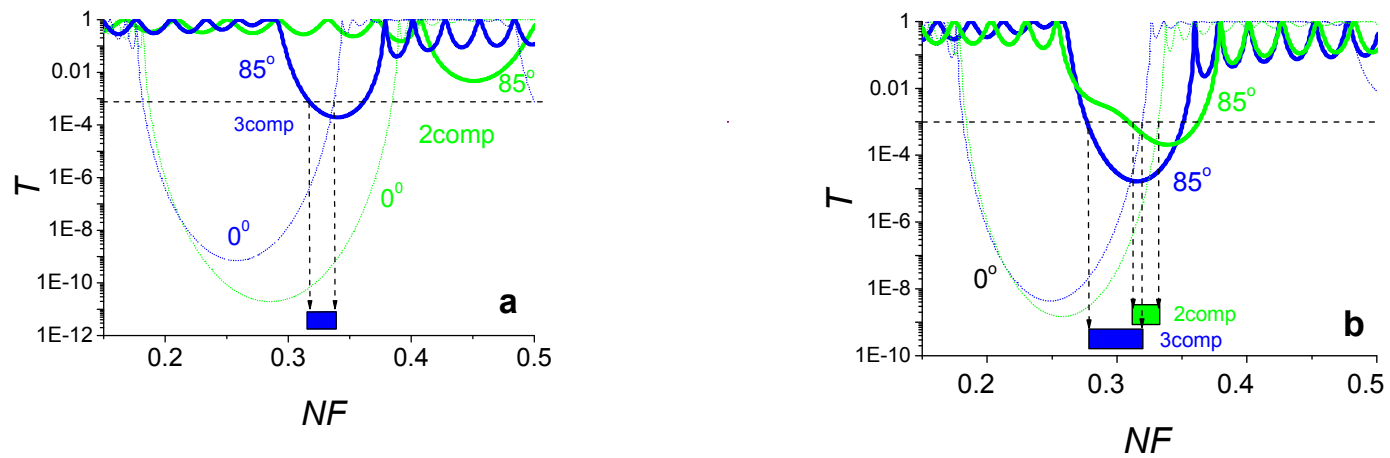

Fig. 4. The ODB's width (filled rectangles) and the transmission spectra $T$ for the first PBGs at incident angles of $0^{\circ}$ and $85^{\circ}$ for two-component PC (denoted as $2 \mathrm{comp}$ ) with optical contrasts (a) 3.42/1 and (b) 3.42/1.3, and three-component PC (denoted as 3comp) with an additional layer $N_{t}=1.5$ and (a) $D_{t}=0.2 A$ and (b) $D_{t}=0.27 \mathrm{~A}$. The limiting filling fraction $\operatorname{Limf}_{\mathrm{Si}}$ value is 0.3 . The dashed line corresponds to the level of $R_{P B G}=0.999$ (or $T_{P B G}=0.001$ ).

This structure does not form an ODB, since there is no overlapping of the PBGs for angles of incidence of 0 and $85^{\circ}$, see the curves corresponding to $0^{\circ}$ and $85^{\circ}$ for the $2 \operatorname{comp}$ case on the plot. Observe that the introduction of a $t$-layer with $D_{t}$ $=0.20 \mathrm{~A}$ results in overlapping of the PBGs and leads to the formation of an ODB area. A similar effect can be observed for the structure with $D_{t}=0.27 \mathrm{~A}$ (see Figs. $4 \mathrm{~b}$ ). However, the degradation in the $T$-bands is observed for an angle of incidence $\theta=85^{\circ}$, but it does not lead to a decrease in the width of the ODB area. On the contrary, the ODB width for the three-component PC is wider than that for the equivalent, two-component PC. We conclude that the introduction of the additional, regular layer into a high optical contrast PC, for example 3.42/1, is equivalent to the substitution of a lowrefractive index component, $L$, a process which results in the appearance of ODBs. 


\section{CONCLUSION}

In this paper, three-component 1D PC structures were investigated by modeling them as two-component PCs with an additional regular $t$-layer. The Gap Map approach and the Transfer Matrix Method were used in order to mathematically describe these PC structures. Introduction of the third component allows variation of the PBG areas of the threecomponent $\mathrm{PC}$ and, therefore, engineering of the optical contrast of the original two-component PC. This can be achieved, not by filling the air channels of a two-component PC, but by selecting either the thickness or refractive index of the third component. Using this approach, omni-directional bands can be obtained in high-contrast Si-air periodic structures.

\section{Acknowledgments}

This work has been supported by the ICGEE Programme (funded by IRCSET, Ireland) and Grants from the Russian Foundation for Basic Research, N08-02-01408 and 09-02-00782. The authors wish to express their appreciation to Dr. Ekaterina Astrova for useful discussions and to Ms. Claire Poinsot for her help with the calculations. Anna Baldycheva acknowledges SPIE Travel Award.

\section{REFERENCES}

[1] Yablonovitch, E., "Inhibited Spontaneous Emission in Solid-State Physics and Electronics," Phys. Rev. Lett. 58(20), 2059-2062 (1987).

[2] John, S., "Strong localization of photons in certain disordered dielectric superlattices," Phys.Rev.Lett., 58(23), 2486-2489 (1987).

[3] Joannopoulos, J.D., Meade, R.D., Winn, R.D., [Photonic Crystals], Princeton University Press, P.184, (1995). Joannopoulos, J.D., Winn, S.G., Meade, R.D., [Photonic Crystals. Molding the Flow of Light], 2nd ed., Princeton University Press, (2008).

[4] Busch, K., Lölkes, S., Wehrspohn, R., Föll, H., [Photonic Crystals. Advances in Design, Fabrication, and Characterization], Weinheim: Wiley-VCH (2004).

[5] Yi, Zh. and Qi, W.,"Properties of photonic bandgap in one-dimensional multicomponent photonic crystal", Optoelectronics Lett., 2(1), 15 (2006).

[6] Glushko, A., Karachevtseva, L., "PBG properties of three-component 2D photonic crystals", Photon. Nanostruct., 4, 141-145 (2006).

[7] Rybin, M.V., Baryshev, A.V., Khanikaev, A.B., Inoue, M., Samusev, K.B., Sel'kin, A.V., Yushin, G. and Limonov, M.F., "Selective manipulation of stop-bands in multi-component photonic crystals: Opals as an example", Phys. Rev. B. 77, 205106 (2008).

[8] Tolmachev, V.A., Baldycheva, A.V., Krutkova, E.Yu., Perova, T.S. and Berwick, K., "Optical characteristics of a one-dimensional photonic crystal with an additional regular layer", Proc. of SPIE, 7390, 739017 (2009).

[9] Tolmachev, V. A., Perova, T. S. and Berwick, K.,"Design criteria and optical characteristics of one-dimensional photonic crystals based on periodically grooved silicon," Appl. Opt., 42, 5679-5683 (2003).

[10] Tolmachev, V.A., Perova, T.S.and Moore, R.A., "Method of construction of composite one-dimensional photonic crystal with extended photonic band gaps," Opt. Express, 13(21), 8433-8441 (2005).

[11] Tolmachev, V., Perova, T., Krutkova, E., Khokhlova, E., "Elaboration of the gap map method for the design and analysis of one-dimensional photonic crystal structures", Physica E: Low-dimensional Systems and Nanostructures, 41, 1122-1126 (2009).

[12]Fink, Y., Winn, J.N., Shanhui, F., Chiping, C., Kimerling, L.C., “A dielectric omni-directional reflector”, IEEE J. Sel. Top. Quantum Electron, 12, 1345 (2006).

[13] Chigrin, D.N., Lavrinenko, A.V., Yarotsky, D.A., Gaponenko, S.V., "Observation of total omnidirectional reflection from a one-dimensional dielectric lattice," Appl.Phys. A, 68, 25-28 (1999).

[14] Russell, P.St.J., Tredwell, S., Roberts, P.J., "Full photonic bandgaps and spontaneous emission control in 1D multilayer dielectric structures," Opt.Commun., 160, 66-71 (1999).

[15] Perova, T.S., Tolmachev, V.A., Astrova, E.V., "Tunable photonic structures based on silicon and liquid crystals", Proc. of SPIE, 6801, 68010W1 (2008). 
[16] Tolmachev, V. A., Astrova, E. V., Pilyugina, Yu. A., Perova, T. S., Moore, R. A. and Vij, J. K. "1D photonic crystal fabricated by wet etching of silicon", Optical Materials, 28(5) 831-835 (2005).

[17] Azzam, R. M. A., Bashara, N. M., [Ellipsometry and polarized light], Amsterdam, North-Holland, P.334 (1977).

[18] Tolmachev, V. A., Astrova, E.V., Perova, T.S., Zharova, J.A, Grudinkin, S.A., Melnikov, V.A., "Electrotunable in-plane one-dimensional photonic structure based on silicon and liquid crystal", App. Phys. Lett., 90, 011908 (2007).

[19] Tolmachev, V. A., Perova, T.S. and Berwick, K., "Design of 1D composite photonic crystals with an extended photonic band gap," J. Appl. Phys. 99, 033507 (2006).

[20] Tolmachev, V. A., Perova, T. S., Ruttle, J. and Khokhlova, E., "Design of One-dimensional Photonic Crystals Using Combination of Band Diagrams and Photonic Gap Map Approaches", J.Appl.Phys. 104, 033536 (2008).

[21] Kim, S. and Hwangbo, C. K., "Design of omnidirectional high reflectors with quarter-wave dielectric stacks for optical telecommunication bands", Appl. Optics, 41(16) 3187-3192 (2002). 